\title{
Effective psychotherapy in a racially and culturally diverse society
}

\author{
Kamaldeep Bhui \& Neil Morgan
}

\begin{abstract}
This article is aimed at psychotherapists and health practitioners who have not reflected on the role of race, culture and ethnicity in the provision of psychological treatments. We highlight the key issues of importance in engagement, assessment and ongoing therapy, supporting practitioners to reach a stage of pre-competency. Competency will be achieved with additional training, supervision and innovation.
\end{abstract}

Psychological treatments are an essential part of a comprehensive mental health service, and there have been a number of influential reviews of their effectiveness. Roth \& Fonagy (2005), for example, concluded that psychodynamic interventions are helpful in borderline states and that family therapy is useful for eating disorders and in the treatment of children and adolescents. Cognitive-behavioural therapy (CBT) is an effective treatment for anxiety, obsessive-compulsive disorder (OCD) and depression, and in the management of hallucinations and delusions in chronic mental illness (Turkington et al, 2006). Expressed emotion work is effective in preventing relapse in schizophrenia (although it is rarely well resourced).

The ability to conduct psychotherapy effectively with racially and ethnically diverse populations is becoming increasingly relevant and is recognised to be important in addressing inequalities, which may also be patterned by differences in age, gender, class and sexual orientation. In this article we focus on race, culture and ethnicity. We will address issues surrounding gender and sexual diversity in a future article.

Regarding the evidence base, few evaluations of the effectiveness of psychotherapy have included adequate numbers of ethnic groups (Alvidrez et al, 1996), and few studies report on adaptations of proven interventions for use by culturally and linguistically unique populations.

In many National Health Service (NHS) psychotherapy departments in the UK large numbers of people are treated, often by a few highly trained senior therapists and by psychiatric, psychology, social work and psychotherapy trainees under supervision. As few training experiences include attention to racial and cultural implications for effective psychotherapy, experienced and trainee therapists alike share the need to develop and adapt their interventions for a society that is increasingly racially and culturally diverse.

Critiques of this subject area tend to emerge from experts in culture, health and illness, and include sociological and anthropological disciplines that use specific methodologies for generation of knowledge. These analyses are often theory rich and not easily accommodated into everyday practice, although they can highlight its limitations. Alternatively, psychotherapists practising in multi-ethnic areas immediately recognise the need for better knowledge and models of work with multi-ethnic populations, but it is difficult to ensure that adaptations of existing psychological interventions are effective. In the absence of both well-established guidelines and an adequate evidence base, we consider here some of the complex issues facing psychotherapists working in multicultural and multiracial populations. The article is aimed at practising psychotherapists rather than researchers in cultural psychiatry or medical anthropology.

\section{Definitions}

The language in which cultural, racial and ethnic issues are described is often used imprecisely and

Kamaldeep Bhui is Professor of Cultural Psychiatry and Epidemiology and Director of the MSc course in transcultural mental healthcare at Barts and the London School of Medicine (Centre for Psychiatry, Wolfson Institute of Preventive Medicine, Charterhouse Square, London EC1A 6BQ, UK. Email: k.s.bhui@qmul.ac.uk) and an honorary consultant psychiatrist in psychotherapy at Mile End Hospital, which is part of the East London and City Mental Health Trust. His interests include racism, cultural identity and religious factors in healing. Neil Morgan is a consultant psychiatrist and Head of Psychotherapy at the Department of Psychotherapy at Mile End Hospital. The Department receives referrals for people from diverse racial and cultural backgrounds in East London. The authors are developing models of improved practice and psychotherapy training processes that produce culturally capable practitioners. 
this leads to much confusion. In Box 1 we offer some working definitions that we have found useful, but acknowledge that these may not capture definitions on which all researchers, social scientists or psychiatrists can agree.

\section{Assessment}

In assessing the mental state of patients, clinicians need to take into account cultural variations of psychopathology and they should be aware of the manner in which empathy and communication can be profoundly influenced by racial and ethnic differences between patient and therapist (Elfenbein \& Ambady, 2002; Bhui \& Bhugra, 2004). Dogra \& Karim (2005) have drawn attention to the possible repercussions of healthcare providers' lack of awareness of cultural differences: lack of knowledge results in inability to recognise the differences; selfprotection and denial lead to an attitude in which such differences are seen as insignificant; and universalistic assumptions are reflected in actions (Box 1).

\section{Box 1 Definitions}

Culture Helman (2000) defines culture as a set of guidelines inherited by members of a particular society that tell them how to view the world, how to experience it emotionally and how to behave in relation to other people. Culture is transmitted by symbol, art, ritual and language.

Ethnicity A community whose heritage offers its members important characteristics in common that make it distinct from other communities is said to have a shared ethnicity - ethnicity is a boundary that distinguishes 'us' from 'them'. It includes an appraisal of appearance, subjective identification, cultural and religious affiliation, and social exclusion (Modood et al, 1997: p. 13).

Race Legal usage: in the Race Relations (Amendment) Act 2000 a racial group is a group of people defined by their race, colour, nationality (including citizenship), or ethnic or national origins. Jews, Sikhs, Gypsies and Irish Travellers have been recognised by the courts as racial groups for the purpose of the Act (http:/ / www. cre.gov.uk/duty/grr/introduction.html)

Popular usage: in biology, the term race distinguishes the distinct populations (subspecies) of a species (including the human species). Many regard race as a social construct. Many think it has genetic basis. The most widely used human racial categories are based on visible traits (especially skin colour, facial features and hair texture), genes and self-identification. Concepts of race, as well as specific racial groupings, vary by culture and over time, and are often controversial, for scientific reasons as well as because of their impact on social identity and 'identity politics' (http://en.wikipedia.org/wiki/Race).

Religion Religious and spiritual beliefs and practices are more common in some ethnic groups than in others. Indeed, some faith groups are defined as distinct not along cultural or ethnic

lines but because of a reliance on religious and spiritual ways of living.

Identity Identity has cultural, ethnic, religious as well as personal components. Cultural, religious and ethnic identity formation and expression are personal processes that include conscious and unconscious compromises between aspired, experienced and imposed identities. Such identities are challenged during the acculturation experience.

Acculturation This refers to the gradual physical, biological, cultural and psychological changes that take place in individuals and groups when contact between two cultural groups takes place. There is pressure on newcomers to conform to and accommodate the dominant cultural lifestyle. Such pressure is handled differently by different individuals, and 'acculturation stress' can create vulnerability to certain health problems as well as susceptibility to increase in psychological symptoms (Loshak, 2003). Berry (2004) defines four states of acculturation: integration (both the individual's original culture and the dominant culture are valued); separation (the individual's culture is valued and the dominant culture is devalued); assimilation (the dominant culture is valued but the individual's culture is devalued; marginalisation (both the individual's culture and the dominant culture are devalued).

Universalism This is an approach to health and social care (in this article, to psychological treatments) that reflects the assumption that, despite specific cultural and historical contexts in which people live their lives, all interventions suit all cultural groups ('one size fits all'). Universalism assumes that assessments, and emergent recommendations and interventions, are universally effective and acceptable. 
However, in a diverse society, specific religious taboos and cultural values may prevent take up of psychological treatment, in favour of more understood or culturally congruent approaches. For example, prayer, rituals and the lay referral system may alleviate mental distress without resort to formal systems of healthcare (Kleinman, 1980). In non-Western cultures, there may not be the same emphasis on talk as a form of treatment, or on introspection, exploration or personal disclosure. Recovery may be expected following biomedical forms of treatment such as injection, medication or physical investigations. More directive instructions may be expected, and the absence of a clear duration for treatment and confidence in expected recovery may puzzle those who expect more paternalistic styles of healthcare. Furthermore, communication between therapist and patient may be affected by different previous experiences of help-seeking and carers, by different notions of selfhood and intimacy, and by culturally determined norms of what therapists, doctors, nurses or social worker are expected to do.

Although these issues are most likely to arise in diverse cultural settings with cultural differences between therapist and patient, they may well also become evident where therapist and patient are from the same ethnic group but belong to different subcultural groups.

In the absence of understanding of each other's expectations, miscommunication can arise and this will affect the patient-therapist relationship. The therapist may not understand why a patient does not adhere to treatment, or perhaps why family decisions and views are as important in healthcare uptake as the decisions of the patient. The patient may end up rejecting the therapist if they feel that the therapist is not able to understand or sustain a thoughtful consideration of the role of culture, race and ethnicity in the recovery process. Akhtar (2006) suggests that the immigrant analyst, and by our inference any therapist, must:

- maintain cultural neutrality toward 'native' patients

- wonder about the patient's motivation for choosing a therapist of different ethnicity

- scan the patient's associations for inter-ethnic clues to deeper transferences

- negotiate the dilemmas posed if analysis is conducted in a language other than one's mother tongue

- avoid shared projections, acculturation gaps and nostalgic collusion.

We propose that these issues are pertinent irrespective of the ethnic match between therapist and patient, or the ethnic origin of the therapist.

\section{Cultural perspectives on the meaning of 'therapy'}

Mental distress is not always seen as a disease or illness: it might be thought of as an outcome of family or interpersonal dysfunction, or of adverse life events and misfortune, or a consequence of breaches in religious and cultural codes of conduct. In culturally diverse societies, practitioners and services will need to adapt interventions, or deliver them with a discerning and sensitive appreciation of the way a treatment is received.

Tseng (1999) classifies therapies around the world into three types: the culturally embedded (e.g. Zar possession and trance states); those influenced by the culture (e.g. Morita and naikan therapies); and those based on cultural elements found in a mainstream model (e.g. CBT).

Many societies practise indigenous therapies, and migrants and ethnic groups often resort to these more familiar forms of healing. Psychotherapies that are unique to specific societies make use of the cultural fabric of that society, with its associated beliefs and aspirations and ways of seeing the world. Mobilising these cultural beliefs can lead to recovery.

Knowledge of culturally embedded or influenced therapies of non-Western origin may be of value in Western settings, where expectations of such therapies may arise among ethnic groups and recent migrants. Furthermore, these therapies include processes of emotional transformation that may not be seen as strictly psychological but may include psychological elements.

In some cases it will be necessary to re-examine the patient's explanatory model of their problems and their previous help-seeking before therapy can begin. The explicit rationale for psychotherapy may need explanation. Compromise and consensus will be required to promote engagement and to nurture a treatment alliance. 'Intercultural therapies' already specifically acknowledge this, and includea mutually respectful exploration of the therapy and of perceived problems and solutions, so that a framework is found within which the therapist and patient can work. With such an approach, psychotherapeutic models such as CBT, psychodynamic approaches and family therapies have the potential to be used effectively across cultures.

\section{Theoretical, technical and philosophical adaptations}

Tseng (2001: pp. 515-595) has outlined the communication issues that must be considered when working across cultures. These include an understanding of 
the mutual expectations of therapist and patient. Existing interventions may be ineffective not only because of miscommunication and divergent health beliefs, but because theoretical and technical failures can culminate in non-adherence. For example, attachment theories in the West emphasise the unique relationship first between mother and child, and then father and child, and then between the child and the outside world. In extended family systems in Eastern societies, there may be less immediate reliance on the mother in the postnatal period, with a number of key figures providing care. For mother this offers practical support, and some physical and emotional relief; for the child it offers multiple attachment figures that are all important for development and are trusted as carers. Of course the level of intimacy may vary: for example, breast-feeding or nappy changing or bathing may be performed by fewer figures. None the less, a theory of attachment that cannot encompass multiple attachments as healthy, as opposed to perceiving these as enmeshed or diffuse or pathological non-attachment to the mother, will need revision. In cross-cultural therapy, theories of psychotherapy will be challenged. If the therapist holds a culturally insensitive theoretical model the patient may fail to engage with or adhere to treatment. Multiple attachments also have implications for development of the self, either as an I-self organised around the individual embodied person or the We-self that reflects a sociocentric self. A sociocentric self is experienced in social groups in which all people are connected, and the self consists of multiple compatible (and not pathological) selves (or self states). Such theoretical differences will determine how technical aspects of the therapy are managed.

Another example of a potential cross-cultural limitation of Western therapeutic theory is its appropriation of the classical Oedipus myth. This finds expression and use in therapeutic processes but is a culturally influenced theory (Bhugra \& Bhui, 2002). The parent-child incest taboo, found in all cultures, institutes the distinction between gender, generations and contact with the external world. In psychoanalytic terms this refers to the triadic Oedipal constellation, but the figures of the triad may vary depending on the culture. For example, in the Indian setting the mother's brother or another 'father' figure may be the rival with whom Oedipal conflicts are resolved; in a matriarchal society this figure may even be female.

\section{Interpreters}

The use of interpreters across cultures has been described in detail in an earlier article is this journal (Farooq \& Fear, 2003). Here we will consider the impact on emotional communications. Using interpreters is an inevitable necessity if services and interventions are to be offered to the linguistic minorities. All information has to be processed through a third person's linguistic-emotional framework before anything is known about the patient. In turn, the patient can learn something of the therapist only through the interpreter. Gestures, smiles and non-verbal behaviour remain important, but steady, slow and carefully taken steps towards mutual understanding are difficult to sustain through an interpreter.

After a lengthier and yet, compared with work with an English speaker, a less revealing process, the therapist may still be faced with theoretical and philosophical uncertainties. Each theoretical concept will need more weighing up and some therapies, for example psychodynamic work, may be impossible, as the necessary level of intimacy may not be easily achieved unless years are dedicated to the task. Even cognitive-behavioural treatments may be undermined if homework and task completion are not understood, or if therapeutic services are seen as part of a medical review process. This is a not uncommon scenario with refugees and asylum seekers, who often find the process of CBT puzzling or anxiety-promoting.

Interpreters may have their own expectations of the situation and understanding of emotional states. They also have different levels of linguistic proficiency. The sensitive timing in the delivery of psychotherapeutic interpretations linking past and present, and patients' disclosure of intimate traumas and precious aspects of self, may all be jeopardised if the interpreter and the therapist are not attuned. Both need to be experienced and perceptive of the quality of their working relationship and their limitations as a professional dyad working with a patient.

Emotional communications across racial and cultural groups are complex and subject to distortion (Bhui \& Bhugra, 2004). Involving a third person in this communicative process may further distort knowledge about the other or, given the limited time available for therapy, leave certain possibilities unexplored. One solution is to ensure that bilingual therapists are recruited and trained to meet the needs of the local population, but this does not overcome the failure of theory or skills in negotiating meanings attached to the therapeutic process.

\section{Racial, ethnic or identity matching}

Ethnic matching has been proposed as a way of addressing cultural differences, including those of race, language and religion. Nevertheless, there is little good empirical evidence that it leads to 
Box 2 Key requirements for racially inclusive psychotherapy

- The therapist needs to be skilled in exploring racial and cultural identity

- The therapistmustunderstand that, for some patients, life events with racial elements are part of a social reality that can become an internal representation and that can emerge in their mental life, fantasy and beliefs

- The therapist must have the capacity to perceive, apprehend and think through the primitive feelings of aggression, hatred, humiliation and shame that accompany racial encounters, conflicts and enactments

(after Carter, 1995)

improved outcomes. It may, however, improve engagement and prevent patients' silent drop out of a treatment that they perceive to be incongruent with their priorities. Shared experience may be important for some patients to enter a therapy: a Black therapist may have had the same experiences of racism, or social exclusion or migration as a Black patient. However, the therapist may still differ in terms of age, gender, educational level and social class.

Just as patients often seek out therapists of the same gender or of a specific age group they may also seek out therapists they perceive to be more able or confident. These may be from a different ethnic group, but perhaps also from their own ethnic group. Thomas (1992) describes these sentiments as pre-transference phenomena fuelled by fantasy, myth and internal representations of the other, and by popular portrayals of ethnic groups in the social environment. Morgan (1998) described how a Black patient sought out a White therapist and (the patient) discovered her own internalised racism and denigration of Blackness. A White therapist was equated with a better therapist.

Issues of confidentiality can also cause patients to seek therapists who are outside of their cultural group. Yet therapists who do not speak the primary language of the patient may not be able to communicate effectively, or conceptualise the patient's distress in words that are meaningful to the patient.

Some patients experience encounters with people from other ethnic groups as traumatic, or as experiences that promote anxiety, fear and primitive impulses that together characterise the dynamics of racial thinking.

Some religious minorities feel that therapy with a non-religious therapist or with a therapist from a different religious group would pose insurmountable obstacles (Lowenthal, 1999).

Language apart, it may not be the ethnic or racial group of the therapist that is crucial. Carter (1995) asserts that the identity status of the therapist and the patient, in terms of the extent of exploration of their own cultural identity, determines the basis of a match or mismatch in therapy. He proposes five stages in the development of a mature cultural identity: separated, pre-encounter, encounter, exploration and closure, in which an identity is finally embraced. Within this process a key discovery that a White therapist has to make is that of the role of 'White privilege'. Carter also identified key skills required by therapists practising racially inclusive therapy, and these are listed in Box 2.

\section{Communication and emotion}

The role of communication across cultures and the difficulties of knowing the other have been discussed in an earlier APT article (Bhui \& Bhugra, 2004). The very act of talking about and putting into words deep personal emotional experience formed from the present and the past results in an alienation of the words from the experience (Nobus, 2000). Communication of internal states of mind is difficult even in a shared language. Across languages and cultures, the task must include slow and paced review of all that is thought to be known and assumed by both the therapist and the patient. Add to this the divergent expectations of what constitutes therapy, especially among recent migrants, and there is a real potential for a failure of communication. This may undermine any modality of therapy, leading to unexplained drop out of treatment and therefore a lack of effectiveness; what Morgan (1998) has called the 'silence' of race.

In Box 3 we make recommendations to support culturally capable practice. These are aimed at developing basic competencies to address some of the very challenging dilemmas that therapists encounter in the consulting room. However, culture, race and ethnicity may be misunderstood to refer to homogeneous groups. Although these recommendations will help to engage patients, it must be remembered that ethnic and racial groups can differ in many other ways, influenced by age, gender, class, sexual orientation, preferred language and degree of integration into British society.

The elements of good practice listed in Box 3 are often those that are presumed to be helpful; for example, they may be the factors that mediate benefit when ethnic matching is used. An important component of working in multiracial, multi-ethnic and multicultural populations is noticing and 
Box 3 Recommendations for culturally capable practice in psychotherapy

Therapists should:

- consider their preconceptions about the race and ethnicity of the patient and of that person's family

- recognise that an individual may be quite different from other members of the same racial and ethnic group

- consider how racial and ethnic differences between themselves and the patient might affect psychotherapy; these include:

- differences in conceptualisation of mental health and illness

- differences in conceptualisation of the self in relation to family and community

- differences in communication styles

- acknowledge that power, privilege and racism might affect interaction with patients

- when in doubt about the importance of race and ethnicity and treatment err on the side of discussion

- keep learning about issues of race and ethnicity, and become a 'mini-ethnographer' with patients and the public

- notice, apprehend and think through the racial transference so that it is understood, acquires therapeutic value and is not reenacted in the consulting room

(adapted from Cardemil \& Battle, 2003)

managing the racial transference. This may include racial material acting as a vehicle for the expression of transference defence, or drive derivatives or object ties (see Moodley \& Palmer, 2006). Racebased transference may also be an expression of intrapsychic conflict, and involve splitting and projective defences; racial material may reveal a person's own experience of racism, and their racial identity as internalised representations of past and present relationships. Irrespective of modality of therapy, such processes should be identified and managed in the service of recovery.

\section{Conclusions}

This article has given a brief outline of some key issues facing therapists working in multicultural and multiracial societies (Bhugra \& Bhui, 1998). There is a valuable literature on this topic, and Box 4 lists some further reading.

\section{Box 4 Further reading}

Cardemil, E. V. \& Battle, C. L. (2003) Guess who's coming to therapy? Getting comfortable with conversations about race and ethnicity in psychotherapy. Professional Psychology: Research and Practice, 34, 278-286.

Darling, L. (2004) Psychoanalytically informed work with interpreters. Psychoanalytic Psychotherapy, 18, 255-267.

Inayat, Q. (2005) Psychotherapy in a multi-ethnic society. Psychotherapist, 26, 7 .

Layard, R. (2006) The case for psychological treatment centres. BMJ, 332, 1030-1032. doi:10.1136/bmj.332. 7548.1030

Neki, J. S. (1975) Guru-chela relationship: the possibility of a therapeutic paradigm. American Journal of Orthopsychiatry, 43, 755-766.

Patel, N., Bennett, E., Dennis, M., et al (2000) Clinical Psychology, Race, and Culture: A Training Manual. British Psychological Society Books.

Prince, R. (1980) Variations in psychotherapy procedures. In Handbook of Cross Cultural Psychopathology (eds T. C. Triandis \& J. G. Draguns), vol 6. Allyn \& Bacon.

Tesone, J. E. (1996) Multi-lingualism, word-presentations, thing-presentations and psychic reality. International Journal of Psychoanalysis, 77, 871-881.

Wheeler, S. (2006) Difference and Diversity in Counselling: Contemporary Psychodynamic Perspectives. Palgrave Macmillan.

We do not propose major technical adaptations of interventions, but suggest that an essential 'precompetency' is to notice the expression of culture and race in therapeutic encounters, and to incorporate these expressions within therapeutic processes and thinking, while taking notice of therapeutic structures that may hinder recovery.

\section{Declaration of interest}

None.

\section{References}

Akhtar, S. (2006) Technical challenges faced by the immigrant analyst. Psychoanalytic Quarterly, 75, 21-43.

Alvidrez, J., Azocar, F. \& Miranda, J. (1996) Demystifying the concept of ethnicity for psychotherapy researchers. Journal of Consulting and Clinical Psychology, 64, 903-908.

Berry, J. W. (2004) Psychology of group relations: cultural and social dimensions. Aviation, Space, and Environmental Medicine, 75 (suppl. 1), C52-C57.

Bhugra, D. \& Bhui, K. (1998) Psychotherapy for ethnic minorities. British Journal of Psychotherapy, 14, 310-326.

Bhugra, D. \& Bhui, K. (2002) Is the Oedipal complex universal? Problems for sexual and relationship psychotherapy across cultures. Sexual and Relationship Therapy, 17, 69-86.

Bhui, K. \& Bhugra, D. (2004) Communication with patients from other cultures: the place of explanatory models. Advances in Psychiatric Treatment, 10, 474-478.

Cardemil, E. V. \& Battle, C. L. (2003) Guess who's coming to therapy? Getting comfortable with conversations about race and ethnicity in psychotherapy. Professional Psychology: Research and Practice, 34, 278-286.

Carter, R. (1995) The Influence of Race and Racial Identity in the Psychotherapy Process: Towards a Racially Inclusive Model. John Wiley \& Sons. 
Dogra, N. \& Karim, K. (2005) Diversity training for psychiatrists Advances in Psychiatric Treatment, 11, 159-167.

Elfenbein, H. A. \& Ambady, N. (2002) On the universality and cultural specificity of emotion recognition: a meta-analysis. Psychological Bulletin, 128, 203-235.

Farooq, S. \& Fear, C. (2003) Working through interpreters. Advances in Psychiatric Treatment, 9, 104-109.

Helman, C. (2000) Culture, Health and Illness. ButterworthHeinemann.

Kleinman, A. (1980) Patients and Healers in the Context of Culture (Thesis). University of California Press.

Loshak, R. (2003) Working with Bangladeshi young women. Psychoanalytic Psychotherapy, 17, 52-67.

Lowenthal, K. (1999) Religious issues and their psychological aspects. In Mental Health Service Provision for a Multicultural Society (eds K. Bhui \& D. Olajide), pp. 54-65. W. B. Saunders.

Modood, T., Berthoud, R., Lakey, J., et al (1997) Ethnic Minorities in Britain. Policy Studies Institute.

Moodley, R. \& Palmer, S. (2006) Race, Culture and Psychotherapy. Routledge.

Morgan, H. (1998) Between fear and blindness: the white therapist and the black patient. Journal of the British Association of Psychotherapists, 34, 48-61.

Nobus, D. (2000) Jacques Lacan and the Freudian Practice of Psychoanalysis. Routledge.

Roth, A. \& Fonagy, P. (2005) What Works for Whom? A Critical Review of Psychotherapy Research (2nd edn). Guilford Press.

Thomas, L. (1992) Racism and psychotherapy. Working with racism in the consulting room: an analytical view. Intercultural Therapy: Themes, Interpretations and Practice (eds J. Kareem \& R. Littlewood). Blackwell Science.

Tseng, W. S. (1999) Culture and psychotherapy: review and practical guidelines. Transcultural Psychiatry, 36, 131-179.

Tseng, W. S. (2001) Handbook of Cultural Psychiatry. Academic Press.

Turkington, D., Kingdon, D. \& Weiden, P. J. (2006) Cognitive behavior therapy for schizophrenia. American Journal of Psychiatry, 163, 365-373.

\section{MCQs}

1 Psychotherapists working with Indian patients will need to be proficient in:

a the patient's first language in order to deliver any therapy

b working with interpreters

c assessing linguistic proficiency and the need for interpreters

d Hindu mythology

e always working through the family elder rather than the patient.

2 Interpreters:

a should provide only literal translations of what is said

b may offer useful information about the cultural background c always offer unbiased and objective translation of the patient's accounts

d should provide detailed information about unconscious process

e are best selected by racial matching with the patient.

3 Ethnic matching:

a is necessary for effective therapy

$\mathrm{b}$ is the same as racial matching

c removes the racial transference

d may be helpful in engagement rather than outcome

e can be done on scientific and objective grounds.

4 Acculturation:

a always results in the total loss of culture of origin

$b$ includes the acquisition of cultural behaviours and attitudes

c is the same as culture shock on arrival in a new country

d is an experience found only among the migrant group and not the hosts

e occurs when moving from city to city in the same country.

5 The racial transference:

a is important only in psychodynamic therapies

$\mathrm{b}$ is managed by consciously avoiding racial stereotyping

c can include hostile feelings and hatred

$\mathrm{d}$ is never based on real experiences of racism

e means that the therapist is racist. 